\title{
Uncompensated metabolic acidosis in Covid-19 patients- treat to prevent intubation
}

\author{
Syed Asad Hasan Rizvi ${ }^{1,2}$, Bushra Syed ${ }^{3}$, Mariam Ghori ${ }^{4}$ \\ 1 Doctor (MBBS), Sindh Infectious Diseases Hospital NIPA, Karachi, Pakistan \\ 2 Dow Medical College, Dow University of Health Sciences, Karachi, Pakistan \\ 3 Undergraduate student (MBBS), Dr. Ruth K.M. Pfau Civil Hospital Karachi, Dow University of Health Sciences, Karachi, Pakistan \\ 4 Undergraduate student (MBBS), Jinnah Postgraduate Medical Centre, Jinnah Sindh Medical University, Karachi, Pakistan \\ *Corresponding Author: Dr. Bushra Syed, Dr. Ruth K.M. Pfau Civil Hospital Karachi, Dow University of Health Sciences, Baba-e- \\ Urdu Road, Karachi, Pakistan \\ Email: bushra_1712@yahoo.com; Tel: +923353042402
}

Received: December 14, 2021; Accepted: December 23, 2021

The COVID-19 pandemic has affected 245,373,039 people globally with a death toll bearing $4,979,421$ deaths to date [1]. It can be a cause of life-threatening metabolic acidosis that merits discussion [2]. Approximately 79.7\% COVID affected individuals suffer acid-base disproportion [2]. According to a study, $2.8 \%$ of this total percentage is attributed to metabolic acidosis with $100 \%$ mortality at the end of follow up [2]. Various risk factors predispose a patient with COVID-19 to metabolic acidosis. SARS-CoV-2 infection is associated with grievous dysregulated inflammation (cytokine storm) involving the lungs [3]. With the engagement of the lung parenchyma, hypoxemia ensues, which increases the risk of fatal metabolic acidosis. Not to forget the role of sepsis in hypoperfusion, eventually inflicting metabolic acidosis. SARS-CoV-2 can directly or via sepsis trigger acute kidney injury giving rise to secondary metabolic acidosis in infected patients. Affected patients are at risk of acute liver failure due to the direct involvement of the liver during active infection which is another cause. Furthermore, in patients with diabetes mellitus who suffer from COVID-19, the use of corticosteroids to treat the infection is a potent inducer of diabetic ketoacidosis that predisposes to metabolic acidosis.

Respiratory alkalosis takes place as compensation for metabolic acidosis [4]. The patient hyperventilates which can result in possible respiratory muscle fatigue. Moreover, the inadequate respiratory response due to muscle fatigue results in respiratory failure [4]. According to a study, 45 out of 140 patients with uncompensated metabolic acidosis eventually needed mechanical ventilation [4]. Hence, metabolic acidosis increases the risk of respiratory failure, which may increase the need for intubation. Our experience at a specialized COVID unit in Karachi has been full of such cases, where many patients suffer from acid-base disproportion, and subsequently deteriorate to undergo intubation and mechanical ventilation.

Unfortunately, patients who are intubated and undergo invasive mechanical ventilation have a poor prognosis, especially in cases of COVID-19 infection. There is a high mortality rate associated with Invasive mechanical ventilation (IMV)- 93.6\% for IMV vs. $66.7 \%$ for non-invasive ventilation (NIMV) [5]. For this reason, we believe that acid-base disproportion and the risk factors associated with it must be urgently addressed in COVID19 infection. Early intervention is crucial to prevent mechanical ventilation. This will avoid the unforeseen crisis and poor prognosis associated with intubation.

Acknowledgments: Not applicable.

Conflict of Interest: None to declare.

Ethics approval: Not applicable.

Funding: None to declare.

Patient consent: Not applicable.

\section{REFERENCES}

1. World Health Organization. WHO Coronavirus (COVID-19) Dashboard. www.covid19. who.int (Accessed on 29/10/2021).

2. Alfano G, Fontana F, Mori G, Giaroni F, Ferrari A, Giovanella S et al. Acid base disorders in patients with COVID-19. Int Urol Nephrol 2021;11:1-6.

3. Chhetri S, Khamis F, Pandak N, Al Khalili H, Said E, Petersen E. A fatal case of COVID-19 due to metabolic acidosis following dysregulate inflammatory response (cytokine storm). IDCases 2020;21:e00829.

4. Daniel SR, Morita SY, Yu M, Dzierba A. Uncompensated metabolic acidosis: an underrecognized risk factor for subsequent intubation requirement. J Trauma 2004;57:993-997.

5. Rahim F, Amin S, Noor M, Bahadur S, Gul H, Mahmood A et al. Mortality of patients with severe COVID-19 in the intensive care unit: an observational study from a major COVID-19 receiving hospital. Cureus 2020;12:e10906. 